\title{
Optimized encapsulation of anthocyanin-rich extract from haskap berries (Lonicera caerulea L.) in calcium-alginate microparticles
}

\author{
Giovana Bonat Celli, Amyl Ghanem and Marianne Su-Ling Brooks* \\ Department of Process Engineering and Applied Science, Dalhousie University, Halifax, NS, Canada
}

Received 9 July 2015; accepted 9 August 2015

\begin{abstract}
.
BACKGROUND: The chemical instability of extracted anthocyanins (ACNs) limits their application and broader use as food colorants and health-promoting functional ingredients. Encapsulation technology can improve ACN stability and widen their potential applications.

OBJECTIVE: The objective of this study was to optimize the microencapsulation of ACNs from haskap berries (Lonicera caerulea $\mathrm{L}$.) in calcium-alginate particles by the extrusion/gelation method.

METHODS: Response Surface Methodology (RSM) by Box-Behnken (BB) design was used for the optimization, followed by the desirability function. Three input variables were evaluated: concentrations of sodium alginate $\left(x_{1}, \mathrm{w} / \mathrm{w} \%\right)$ and calcium chloride $\left(x_{2}, \mathrm{w} / \mathrm{v} \%\right)$, and gelation time $\left(x_{3}, \mathrm{~min}\right)$. The responses were encapsulation efficiency $\left(y_{1}, \%\right)$ and particle size $\left(y_{2}\right.$, $\mu \mathrm{m})$.

RESULTS: There was a good fit for the model where encapsulation efficiency was used as a separate response $\left(R^{2}=97.98 \%\right)$, however, the model for particle size did not give as good an agreement $\left(R^{2}=63.86 \%\right)$. The desirability function was used to optimize the two responses simultaneously and the optimum conditions were determined as $9.0 \%(\mathrm{w} / \mathrm{w})$ alginate solution, $2.0 \%(\mathrm{w} / \mathrm{v}) \mathrm{CaCl}_{2}$, and $10 \mathrm{~min}$ in the gelation solution.

CONCLUSIONS: These results illustrate the application of RSM followed by a desirability function to optimize encapsulation parameters for a combined response, where several measures are considered.
\end{abstract}

Keywords: Gelation, Response Surface Methodology, Box-Behnken design, encapsulation efficiency, size

\section{Introduction}

Anthocyanins (ACNs) are members of the flavonoid class of secondary metabolites and the most important water-soluble pigments in higher plants, conferring red, violet, and blue colors to flowers and fruits [1]. In addition to their colorant properties, several studies have related the consumption of these compounds to health benefits [2-4], including the reduction of cancer [5], improved hyperglycemia and insulin resistance [6, 7], reduction of bone loss in ovariectomized rat model [8], and neurocognitive benefits [9]. In this context, haskap berries (Lonicera caerulea L.) have gained the attention of the scientific community due to their high levels of

\footnotetext{
*Corresponding author: Marianne Su-Ling Brooks, Department of Process Engineering and Applied Science, Dalhousie University, PO Box 15000, Halifax, B3H 4R2 NS, Canada. Tel.: +1 902494 6482; E-mail: Su-Ling.Brooks@ dal.ca.
} 
ACNs and potential antioxidant activity $[10,11]$. These fruits are native to northeastern Asia, where they are recognized as the elixir of life by Japanese aborigines, and were recently introduced in the North America market [12]. Different health-related benefits have been associated with these berries using in vitro and in vivo studies [10], providing scientific basis for the development of value-added products.

Despite their functionality, the incorporation of ACNs in different food matrices is limited by their stability once extracted due to their electron deficiency [13], which is often affected by environmental conditions, such as $\mathrm{pH}$ [14], temperature [15], oxygen [16], light [17], heat [18], water activity [19], and presence of other compounds (e.g., phenolic compounds, enzymes, metal ions, sugars, and ascorbic acid) [20]. In this respect, encapsulation is a versatile technology that can address these needs, contributing to the development of high-value food products $[21,22]$.

Encapsulation is a general term to describe any process used to entrap one substance (termed core material or active agent) within another (coating, shell or carrier/wall material) [23]. Encapsulation techniques can extend a product's shelf-life [24], protecting the active components against degradation during storage [25] and maintaining their functionality [26]. They can also help to mask unwanted flavors [27] and increase the effectiveness of natural functional compounds that normally have a lower potency at equivalent levels in comparison to synthetic ingredients [28]. For instance, there is an increasing demand for natural antioxidants that could substitute the synthetic butylated hydroxyanisole (BHA), butylated hydroxytoluene (BHT), and tertiary-butyl hydroquinone (TBHQ). However, some natural candidates are not as efficient as the synthetic products when added at the same levels [29].

The aim of this work was to optimize the encapsulation of an ACN-rich haskap berry extract in calcium-alginate microparticles by the extrusion/gelation method [30]. Firstly, the most appropriate procedure for the preparation of the ACN extract was determined by comparing extraction under stirring conditions with maceration. Then, statistical optimizations of the extrusion/gelation process parameters (i.e. sodium alginate and calcium chloride concentrations and gelation time) using individual responses (encapsulation efficiency and particle size) were conducted by Response Surface Methodology (RSM) using Box-Behnken design. This was followed by applying a desirability function to obtain the optimal conditions for the two responses simultaneously.

\section{Material and methods}

\subsection{Plant material}

Haskap berries (variety Indigo Gem) were used in this study. Berries were mechanically harvested at the Northern Light Orchards (Saskatchewan, Canada) and shipped frozen to LaHave Natural Farms, in Nova Scotia. The berries were then transferred to Dalhousie University and kept at $-35^{\circ} \mathrm{C}$ prior to freeze-drying. Frozen berries were cut in halves and freeze-dried for approximately three days in a Labconco FreeZone 4.5 L Bench-top Freeze Dry System (Labconco, Kansas City, MO, USA) (collector temperature was set at $-85^{\circ} \mathrm{C}$ and pressure was below $0.100 \mathrm{mbar}$ ) (moisture $<5 \%$ ). The freeze-dried samples were stored in desiccator at $-18^{\circ} \mathrm{C}$ until use.

\subsection{Chemicals}

Reagent ethanol, formic and acetic acid, and calcium chloride were purchased from Fisher Scientific (Ottawa, ON, Canada). Alginate Protanal LFR5/60 was kindly donated by FMC BioPolymer (Philadelphia, PA, USA).

\subsection{Assessment of the extraction procedure}

The total ACN content obtained from extraction under stirring conditions was compared with maceration using modified methods described by Musa et al. [31], to determine the most appropriate method for preparing the 
extract. Initial results indicated that there was no significant difference in the extraction yield using freeze-dried berries cut in halves or ground dried berries (particle size $<0.5 \mathrm{~mm}$ ), possibly due to the delicate structure of these berries [12], thus for convenience, freeze-dried berries were used in the extraction without further processing. For extraction under stirring conditions, the freeze-dried samples were combined with $80 \%$ ethanol acidified with $0.5 \%$ formic acid (using a ratio of $1: 25, \mathrm{w} / \mathrm{v}$ solid:liquid) and stirred at $600 \mathrm{rpm}$ for $10 \mathrm{~min}$ at room temperature using a magnetic-stirrer. For maceration, $80 \%$ ethanol acidified with $0.5 \%$ formic acid was added to the freezedried berries (same ratio as under stirring conditions) and left for $2 \mathrm{~h}$ at room temperature, in the absence of light. In both cases, the mixture was filtered through a Whatman filter paper $n^{\circ} 1$ after extraction and kept in a conventional freezer $\left(a t-18^{\circ} \mathrm{C}\right.$ ) in the absence of light until quantification of the total ACN content. The means of the methods were compared by the unpaired $t$-test using SPSS Statistics version 22 software (IBM, Chicago, IL, USA).

\subsection{Microencapsulation of anthocyanin-rich extract}

Prior to encapsulation, the organic solvent in the extract was removed by a rotary evaporator model BM 400 (Yamato Scientific America Inc., Santa Clara, CA, USA) with a water bath kept at $T<50^{\circ} \mathrm{C}$, equipped with a vacuum pump V-700 (Büchi Labortechnik AG, Flawil, Switerzland) and a water circulation cooler WK 230 (Lauda, Lauda-Königshofen, Germany). The resultant aqueous fraction was kept at $-18^{\circ} \mathrm{C}$ protected from light until further use.

A system consisting of a mini-pump with variable flow rate (Fisher Scientific) and a $23 \mathrm{G}$ needle was used for the encapsulation of ACN-rich extract in calcium-alginate microparticles by the extrusion/gelation method (Fig. 1) adapted from Santos et al. [32]. The pH of the aqueous fraction was low (approximately 2.9) and its adjustment was essential to prevent immediate gelation of the alginate solution and precipitation of alginic acid. The $\mathrm{pH}$ of the extract was thus adjusted to approximately 4.5 with $0.1 \mathrm{~N} \mathrm{NaOH}$ prior to mixing with the alginate solution. The ratio extract:alginate solution was set at 1:4 (v/v) based on preliminary results, taking into account the concentration of ACNs in the evaporated extract. The extract/alginate solution was then extruded through the needle into $30 \mathrm{~mL}$ of $\mathrm{CaCl}_{2}$ gelation medium, using an average flow rate of $0.3 \mathrm{~mL} / \mathrm{min}$. The distance between the tip of the needle and the gelation medium surface was fixed at $10 \mathrm{~cm}$. The alginate membrane formed immediately

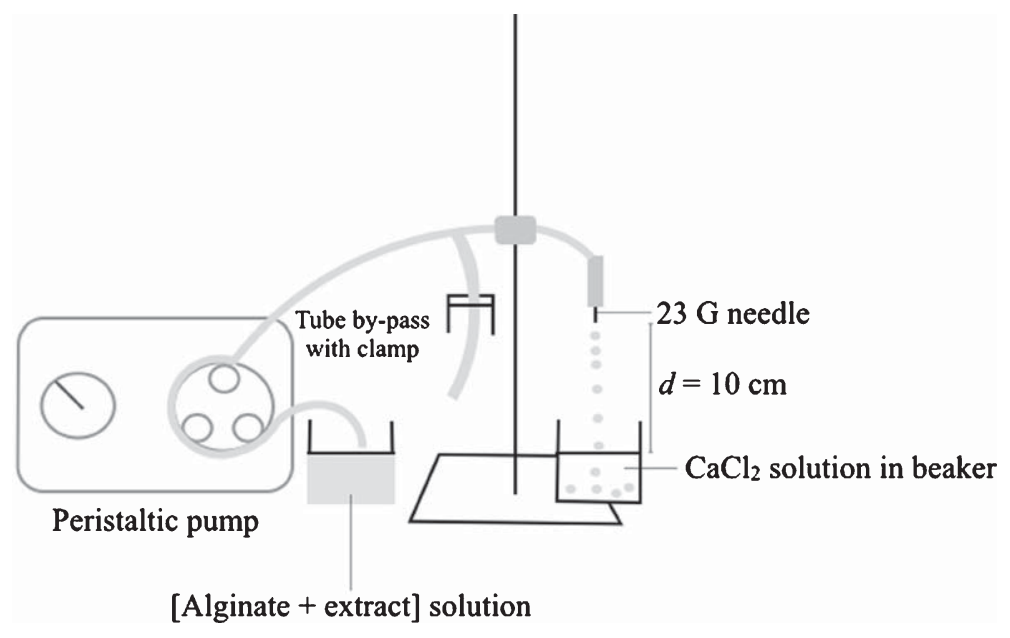

Fig. 1. Diagram of the system used for the preparation of alginate microparticles. A tube by-pass with clamp was used for further adjustment of the flow-rate. 
upon contact of the solution with the $\mathrm{CaCl}_{2}$ medium. After the gelation time, particles were recovered by vacuum filtration, left for $1 \mathrm{~min}$ in $10 \mathrm{~mL}$ of distilled water, and then dried overnight in the dark at room temperature.

\subsection{Experimental design and data analysis}

\subsubsection{Box-Behnken design for optimization of encapsulation}

A Box-Behnken design with three levels $(-1,0,+1$, representing low, middle, and high levels) was used to optimize the encapsulation of ACN-rich extract from haskap berries. The following variables and levels were considered in this study, based on previous results and reports from the literature $[32,33]$ : sodium alginate concentration $\left(\xi_{1}\right)$, calcium chloride concentration acidified with $2 \%(\mathrm{v} / \mathrm{v})$ acetic acid glacial $\left(\xi_{2},\right)$, and time in the gelation solution $\left(\xi_{3}\right)$ (Table 1).

The optimization consisted of 15 runs (with triplicate in the center point) and the design matrix is presented in Table 2. The responses assessed were encapsulation efficiency $\left(y_{1}, \%\right)$ and size of the dried particles $\left(y_{2}, \mu \mathrm{m}\right)$. Design-Expert ${ }^{\circledR}$ version 9.0 software (Stat-Ease, Inc., Minneapolis, MN, USA) was used for analysis and mod-

Table 1

Input variables (natural and coded) and levels chosen for screening

\begin{tabular}{|c|c|c|c|c|}
\hline \multirow[t]{2}{*}{ Input variable } & \multirow[t]{2}{*}{ Coded variable } & \multicolumn{3}{|c|}{ Levels } \\
\hline & & -1 & 0 & +1 \\
\hline Sodium alginate concentration $(\% \mathrm{w} / \mathrm{w})\left(\xi_{1}\right)$ & $x_{1}=\left(\xi_{1}-9.5\right) / 0.5$ & 9 & 9.5 & 10 \\
\hline $\mathrm{CaCl}_{2}$ concentration $(\% \mathrm{w} / \mathrm{v})\left(\xi_{2}\right)$ & $x_{2}=\left(\xi_{2}-2.5\right) / 0.5$ & 2 & 2.5 & 3 \\
\hline Gelation time $(\min )\left(\xi_{3}\right)$ & $x_{3}=\left(\xi_{3}-20\right) / 10$ & 10 & 20 & 30 \\
\hline
\end{tabular}

Table 2

Uncoded Box-Behnken design matrix with experimental results

\begin{tabular}{lrllll}
\hline No. $^{\text {a }}$ & $x_{1}$ & $x_{2}$ & $x_{3}$ & $y_{1}$ & $y_{2}$ \\
\hline 1 & 9.0 & 2.0 & 20 & 42.25 & $1,119.5$ \\
2 & 10.0 & 2.0 & 20 & 46.20 & $1,125.1$ \\
3 & 9.0 & 3.0 & 20 & 17.97 & $1,119.6$ \\
4 & 10.0 & 3.0 & 20 & 50.66 & $1,129.3$ \\
5 & 9.0 & 2.5 & 10 & 57.84 & $1,113.5$ \\
6 & 10.0 & 2.5 & 10 & 63.12 & $1,123.6$ \\
7 & 9.0 & 2.5 & 30 & 39.39 & $1,120.2$ \\
8 & 10.0 & 2.5 & 30 & 62.27 & $1,121.9$ \\
9 & 9.5 & 2.0 & 10 & 51.85 & $1,118.5$ \\
10 & 9.5 & 3.0 & 10 & 46.50 & $1,124.3$ \\
11 & 9.5 & 2.0 & 30 & 36.79 & $1,115.8$ \\
12 & 9.5 & 3.0 & 30 & 32.32 & $1,114.6$ \\
13 & 9.5 & 2.5 & 20 & 30.57 & $1,121.3$ \\
14 & 9.5 & 2.5 & 20 & 27.90 & $1,126.1$ \\
15 & 9.5 & 2.5 & 20 & 29.33 & $1,125.2$ \\
\hline
\end{tabular}

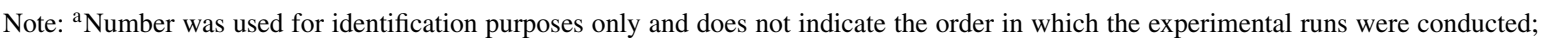
$x_{1}$ - sodium alginate concentration $(\%) ; x_{2}-\mathrm{CaCl}_{2}$ concentration $(\%) ; x_{3}$ - gelation time (min); $y_{1}$ - encapsulation efficiency $(\%) ; y_{2}-$ size $(\mu \mathrm{m})$. 
eling of the responses. A second-degree polynomial equation was fitted for each response variable, generically expressed as:

$$
y=\beta_{0}+\sum_{i=1}^{3} \beta_{i} x_{i}+\sum_{i=1}^{3} \beta_{i i} x_{i}^{2}+\sum_{i} \sum_{j=i+1} \beta_{i j} x_{i} x_{j}
$$

where $y$ is the response; $\beta_{0}$ is the constant (or model intercept); $\beta_{i}$ is the linear coefficient of the coded variables; $\beta_{i i}$ is the quadratic coefficient; $\beta_{i j}$ the cross-product coefficient; $x_{i}$ and $x_{j}$ are the coded independent variables.

\subsubsection{Desirability function}

The desirability function approach [34] was used to optimize the responses simultaneously and to identify the ideal conditions for the preparation of ACN encapsulates. This function involved two phases: (a) each response was transformed into a desirable value $\left(d_{i}\right)$, which can assume values from 0 to 1 ; and (b) based on the individual desirability values, the overall desirability function $(D)$ for all dependent variables was determined.

The following scenarios were considered when maximizing encapsulation efficiency and minimizing size [34]:

Maximization (for encapsulation efficiency):

$$
d_{i}=\left\{\begin{array}{cc}
0 & y_{i} \leq y_{i, \min } \\
{\left[\frac{y_{i}-y_{i, \min }}{y_{i, \max }-y_{i, \min }}\right]^{r}} & y_{i, \min }<y_{i}<y_{i, \max } \\
1 & y_{i} \geq y_{i, \max }
\end{array} \quad \text { for } i=1,2, \ldots, \mathrm{k}\right.
$$

Consequently,

$$
\begin{array}{ll}
d_{i}=0 & \text { response is smaller than the minimum value } \\
0 \leq d_{i} \leq 1 & \text { response is between minimum and maximum values } \\
d_{i}=1 & \text { response is greater than the maximum value }
\end{array}
$$

\section{Minimization (for size):}

$$
d_{i}=\left\{\begin{array}{cc}
1 & y_{i} \leq y_{i, \min } \\
{\left[\frac{y_{i}-y_{i, \max }}{y_{i, \min }-y_{i, \max }}\right]^{r}} & y_{i, \min }<y_{i}<y_{i, \max } \\
0 & y_{i} \geq y_{i, \max }
\end{array}\right.
$$

Consequently,

$$
\begin{array}{ll}
d_{i}=0 & \text { response is greater than the maximum value } \\
0 \leq d_{i} \leq 1 & \text { response is between minimum and maximum values } \\
d_{i}=1 & \text { response is smaller than the minimum value }
\end{array}
$$

where $y_{i, \min }$ and $y_{i, \max }$ are the minimum and maximum acceptable values of the response $\left(y_{i}\right)$, respectively, and $r$ is the weight factor that determines the shape of the desirability graph. The $r$ and importance $(w)$ values were set at 1 and 3, respectively, where the importance refers to the effect of each response on the overall desirability.

Once the $d_{i}$ values were found, the calculation of their geometric mean resulted in the overall desirability $(D)$, as follows:

$$
D=\left(d_{1}^{w_{1}} x d_{2}^{w_{2}} x \ldots d_{k}^{w_{k}}\right)^{1 / \sum_{i}^{k} w_{i}}
$$

where $k$ is the number of variables being optimized. 


\subsubsection{Responses assessed}

Encapsulation efficiency or EE $\left(y_{1}, \%\right)$ was indirectly assessed by measuring the total anthocyanin content in the recovered $\mathrm{CaCl}_{2}$ solution (i.e. amount not entrapped) by the $\mathrm{pH}$ differential method [35]. Absorbance values were measured at $\lambda_{\max }$ and $700 \mathrm{~nm}$ against distilled water using a Genesys $10 \mathrm{~S}$ UV-Vis spectrophotometer (Thermo Scientific, Madison, WI, USA). These analyses were performed in triplicate. As the particles were not completely spherical, the size of the dry particles $\left(y_{2}, \mu \mathrm{m}\right)$ was determined by measuring the major axis of individual particles using a caliper ruler and reporting the average measurement from 10 particles.

\section{Results and discussion}

Two methods for extraction were evaluated in this study. Extraction under stirring and by maceration at room temperature yielded $20.74 \pm 1.07$ and $18.09 \pm 1.19 \mathrm{mg}$ cyanidin 3 -glucoside equivalents/g of dried berries by the $\mathrm{pH}$-differential method. No significant difference was observed between the methods in the unpaired $t$-test $(p>0.05)$. Reports in the literature have indicated significant differences between similar extraction methods. For example, Musa et al. [31] found that stirring guava fruit with solvent for $1 \mathrm{~h}$ at $1,000 \mathrm{rpm}$ resulted in significantly higher antioxidant activity than maceration for 1 and 2 days. Adjé et al. [36] also showed that maceration under stirring resulted in higher recovery of polyphenols and ACNs from Delonix regia tree flower than maceration alone. However, this is very much determined by the range of extraction parameters used, and it was not the aim of this study to optimize the extraction procedure. For this reason, the stirring method was selected for the preparation of the extract for encapsulation as similar ACN recovery was obtained in a shorter period of time.

Alginate particles are commonly formed by extruding a solution of sodium or potassium alginate through a needle or nozzle into a gelation solution containing calcium ions [37]. This is a mild encapsulation method that could contribute to the retention and delivery of ACNs in food products. According to the manufacturer, the sodium alginate used in this study contained approximately $65-75 \%$ of guluronic acid groups. Martinsen et al. [38] showed that particles with highest mechanical strength, lowest shrinkage, and highest porosity were obtained with alginate containing more than $70 \%$ of guluronic acid. An example of the particles produced is presented in Fig. 2.

The ANOVA results showing the significant variables $(p<0.05)$ for the encapsulation efficiency and size of ACNs in calcium-alginate particles analyzed by RSM are presented in Tables 3 and 4, respectively. The results indicated that the models were significant for the responses analyzed $\left(F_{E E}=48.39\right.$ and $\left.F_{\text {Size }}=6.48, p<0.05\right)$. The non-significant lack-of-fit $(p>0.05)$ for both models also indicated that they fitted the experimental data. The

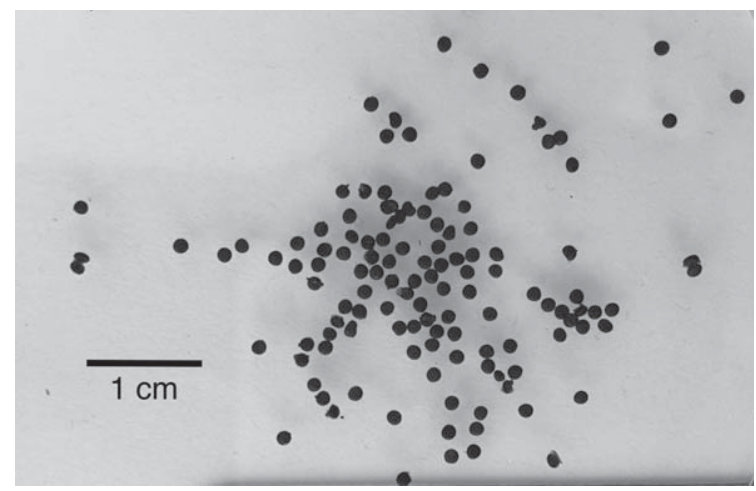

Fig. 2. An example of the particles produced during the optimization after being left to dry overnight at room temperature in the absence of light. 
Table 3

ANOVA results for the model with encapsulation efficiency $\left(y_{1}\right)$ as the response

\begin{tabular}{|c|c|c|c|c|c|c|}
\hline Source & $d f$ & SS & MS & $F$-value & $p$-value & $P(\%)$ \\
\hline Model & 7 & 2460.46 & 351.49 & 48.39 & $<0.0001$ & 97.98 \\
\hline$x_{1}$ & 1 & 525.07 & 525.07 & 72.29 & $<0.0001$ & 20.91 \\
\hline$x_{2}$ & 1 & 109.78 & 109.78 & 15.11 & 0.0060 & 4.37 \\
\hline$x_{3}$ & 1 & 294.37 & 294.37 & 40.53 & 0.0004 & 11.72 \\
\hline$x_{1} x_{2}$ & 1 & 206.42 & 206.42 & 28.42 & 0.0011 & 8.22 \\
\hline$x_{1} x_{3}$ & 1 & 77.42 & 77.42 & 10.66 & 0.0138 & 3.08 \\
\hline$x_{1}^{2}$ & 1 & 538.72 & 538.72 & 74.17 & $<0.0001$ & 21.45 \\
\hline$x_{3}{ }^{2}$ & 1 & 795.86 & 795.86 & 109.57 & $<0.0001$ & 31.69 \\
\hline Error & 7 & 50.84 & 7.26 & & & 2.02 \\
\hline Lack-of-fit & 5 & 47.27 & 9.45 & 5.29 & 0.1665 & 1.8 \\
\hline Pure error & 2 & 3.57 & 1.79 & & & 0.14 \\
\hline Total & 14 & 2511.30 & & & & 100.00 \\
\hline$S$ & 2.70 & & Adjusted $R^{2}$ & 0.9595 & & \\
\hline$R^{2}$ & 0.9798 & & Predicted $R^{2}$ & 0.8590 & & \\
\hline
\end{tabular}

Note: $x_{1}$ - alginate concentration; $x_{2}-\mathrm{CaCl}_{2}$ concentration; $x_{3}$ - time in the gelation solution; $P$ - percentage contributions; $S$ - standard error of the regression; $R^{2}$ - regression coefficient.

Table 4

ANOVA results for the model with particle size $\left(y_{2}\right)$ as the response

\begin{tabular}{|c|c|c|c|c|c|c|}
\hline Source & $d f$ & SS & MS & $F$-value & $p$-value & $P(\%)$ \\
\hline Model & 3 & 180.37 & 60.12 & 6.48 & 0.0087 & 63.86 \\
\hline$x_{1}$ & 1 & 91.80 & 91.80 & 9.89 & 0.0093 & 32.50 \\
\hline$x_{3}$ & 1 & 6.84 & 6.84 & 0.74 & 0.4087 & 2.42 \\
\hline$x_{3}^{2}$ & 1 & 81.72 & 81.72 & 8.81 & 0.0128 & 28.93 \\
\hline Error & 11 & 102.07 & 9.28 & & & 36.14 \\
\hline Lack-of-fit & 9 & 89.05 & 9.89 & 1.52 & 0.4589 & 31.53 \\
\hline Pure error & 2 & 13.02 & 6.51 & & & 4.61 \\
\hline Total & 14 & 282.43 & & & & 100.00 \\
\hline$S$ & 3.05 & & Adjusted $R^{2}$ & 0.5401 & & \\
\hline$R^{2}$ & 0.6386 & & Predicted $R^{2}$ & 0.2650 & & \\
\hline
\end{tabular}

Note: $x_{1}$ - alginate concentration; $x_{2}-\mathrm{CaCl}_{2}$ concentration; $x_{3}$ - time in the gelation solution; $P$ - percentage contributions; $S$ - standard error of the regression; $R^{2}$ - regression coefficient.

models explained 97.98 and $63.86 \%$ of the variations in EE and particle size, respectively. The model for size was not as good as the one for EE (low $R^{2}$ and large discrepancies between adjusted and predicted $R^{2}$ ); however, the coefficients of variation (CV\% $=6.37$ and 2.51 for $\mathrm{EE}$ and size, respectively) indicated an acceptable level of precision and reliability of the models.

In relation to the terms in the model, $x_{3}$ had to be included in the model for particle size for hierarchical purposes (Table 4). The percentage contribution $(P)$ was calculated for all terms by dividing the sum of squares $(S S)$ of each component by the total $S S$ and these results indicated that $x_{1}, x_{1}^{2}$, and $x_{3}{ }^{2}$ affected the encapsulation efficiency by 20.91, 21.45, and 31.6\%. In the case of size, the variables $x_{1}$ and $x_{3}{ }^{2}$ affected 32.50 and $28.93 \%$, respectively. 
The quadratic models for each response variable are described in coded and natural variables, as follows:

$$
\begin{gathered}
y_{1}=28.10+8.10 x_{1}-3.70 x_{2}-6.07 x_{3}+7.18 x_{1} x_{2}+4.40 x_{1} x_{3}+12.04 x_{1}^{2}+14.64 x_{3}^{2} \\
E E(\%)=5160.62-988.52 \xi_{1}-280.39 \xi_{2}-14.82 \xi_{3}+28.73 \xi_{1} \xi_{2}+0.88 \xi_{1} \xi_{3}+48.17 \xi_{1}^{2}+0.15 \xi_{3}^{2} \\
y_{2}=1123.73+3.39 x_{1}-0.92 x_{3}-4.68 x_{3}^{2} \\
\text { Size }(\mu \mathrm{m})=1042.50+6.77 \xi_{1}+1.78 \xi_{3}-0.05 \xi_{3}^{2}
\end{gathered}
$$

The surface graphs for the responses evaluated in relation to the variables presented in the model are illustrated in Fig. 3. The encapsulation efficiency ranged from 17.97 to $63.12 \%$. Figure 3A shows that higher encapsulation efficiency $(63.12 \%)$ could be obtained with a high concentrations of alginate $(10 \%, \mathrm{w} / \mathrm{w})$ and shorter period of time $(10 \mathrm{~min})$ in the crosslinking solution, keeping the concentration of $\mathrm{CaCl}_{2}$ in its center point $(2.5 \%$, w/v) . When the concentration of alginate was decreased to $9 \%(\mathrm{w} / \mathrm{w})$ (keeping the other variables constant), the EE dropped considerably (17.97\%), providing evidence that this variable played an important role in EE. This was also demonstrated by Lotfipour et al. [39], who reported a significant effect of alginate concentration on the EE of probiotics using the extrusion method, whereas the concentration of $\mathrm{CaCl}_{2}$ and gelation time were considered insignificant. A higher concentration of alginate could have blocked the diffusion of encapsulated ACNs due to a denser and thicker membrane with greater availability of active sites for crosslinking with calcium [40]; however, this would be dependent on the concentration of calcium ions and their availability to crosslink the
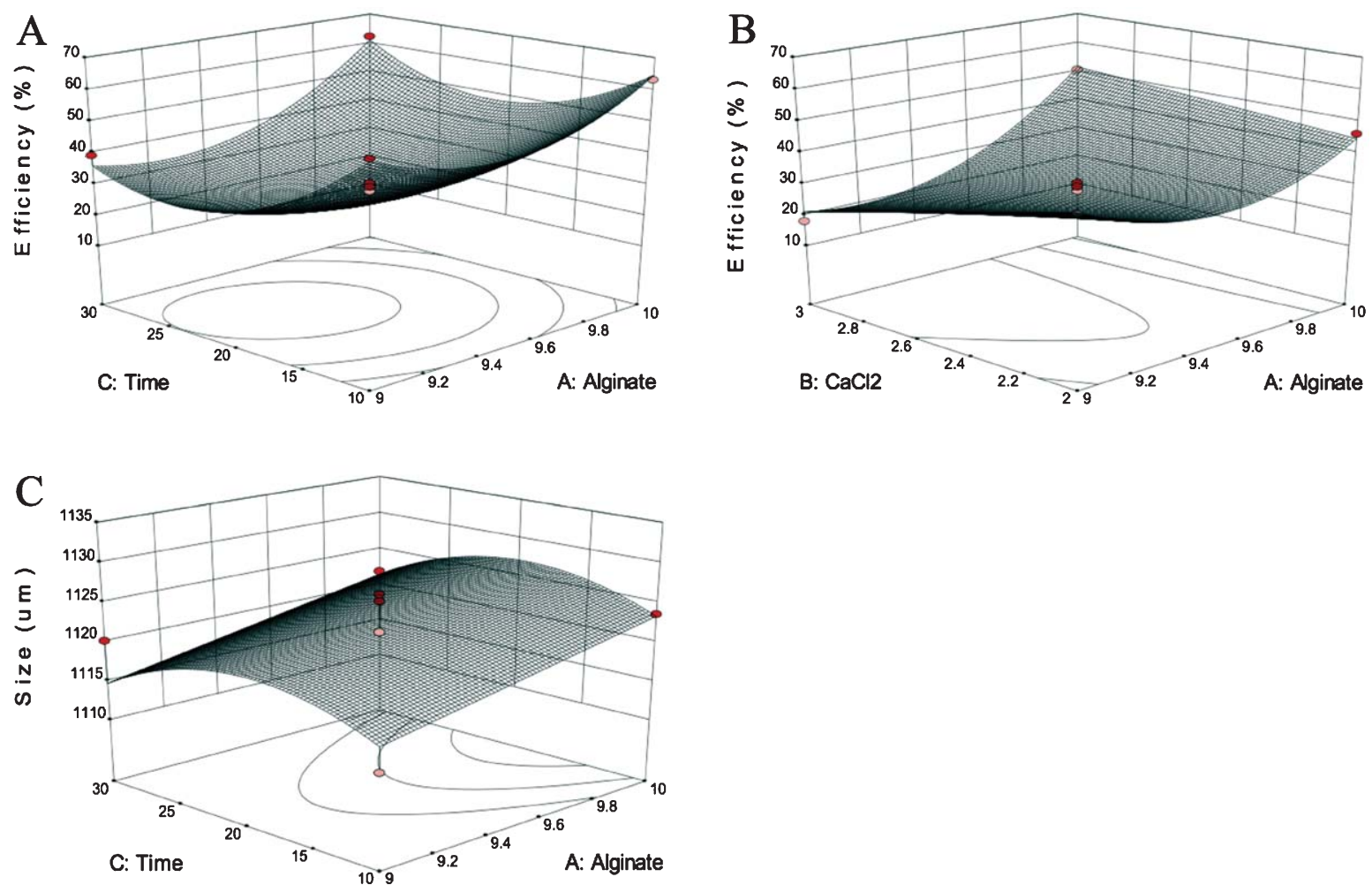

Fig. 3. Surface plots of the two responses assessed (A and B - encapsulation efficiency; C - size) versus the variables included in the model. 
alginate chains and also the time the particles remained in the gelation medium. ACNs are hydrophilic compounds and it is possible that they could have diffused out from the calcium-alginate particles if they remained for too long in the $\mathrm{CaCl}_{2}$ solution.

At a low concentration of alginate $(9 \%, \mathrm{w} / \mathrm{w})$, an increase of $\mathrm{CaCl}_{2}$ concentration from 2 to $3 \%(\mathrm{w} / \mathrm{v})$ resulted in a reduction of EE from 42.25 to $17.97 \%$. The gelation was carried out under an acidic gelling environment to prevent the degradation of ACNs, and the flavylium cation was most likely the $\mathrm{ACN}$ form found during encapsulation $[1,41]$. It could have been possible that higher concentrations of $\mathrm{CaCl}_{2}$ could have led to an electrostatic repulsion and attraction with calcium and chlorine ions, respectively, which could have also contributed to the leakage of anthocyanins from the particles. Jiang et al. [42] also showed that higher concentrations of $\mathrm{CaCl}_{2}$ resulted in lower encapsulation efficiency of $\beta$-glucuronidase in alginate particles.

In relation to size, larger particles were obtained with higher concentrations of alginate $(10 \%, \mathrm{w} / \mathrm{w})$ around the center point for time $(20 \mathrm{~min})$. The concentration of alginate is intimately related to the viscosity of the mixture $[43,44]$ and the behavior during extrusion. Even though viscosity was not evaluated in this study, a subjective assessment of the solutions has indicated a positive correlation between polymer concentration and viscosity. A more viscous solution would reduce the flux and increase the detachment time of droplets from the needle using the same flow rate and pressure, resulting in larger particles. Lotfipour et al. [39] also showed that the concentration of alginate had a significant effect on the particles produced by extrusion. It is likely that the duration of the particles in the gelling solution would impact the formation of the internal network structure within the alginate gel and, consequently, affect the mechanical resistance of the particles, although it was not assessed in this work. Deladino et al. [45] showed that dried calcium-alginate particles prepared with yerba mate extract had a spherical disc shape with a collapsed center, resembling a red blood cell. In this study, the particles also had a disc shape, but no collapse was observed. This collapse during drying has been associated with the heterogeneous gelation mechanism where a dense surface layer and loose core would be observed [45, 46], which was not the case in the present study.

In order to optimize the two responses simultaneously, the desirability function methodology was used in which encapsulation efficiency was maximized, whereas size was minimized. The individual desirability functions for encapsulation efficiency and size were approximately 1.00 and 0.81 , respectively. The overall desirability $(D)$ was approximately 0.90 , with predicted encapsulation efficiency of $68.03 \%$ and size of $1,116.6 \mu \mathrm{m}$. Using this strategy, the optimization of the two responses was predicted at the following conditions: alginate concentration of $9.0 \%(\mathrm{w} / \mathrm{w}), \mathrm{CaCl}_{2}$ concentration of $2.0 \%(\mathrm{w} / \mathrm{v})$, and $10 \mathrm{~min}$ in the gelation solution.

\section{Conclusion}

The encapsulation of ACN-rich extract from haskap berries (Lonicera caerulea $\mathrm{L}$.) in calcium-alginate particles was optimized by RSM using Box-Behnken design. Three input variables (alginate and $\mathrm{CaCl}_{2}$ concentrations, and time for crosslinking) were investigated and their impacts on encapsulation efficiency and size were assessed. Using the desirability function, it was possible to optimize the two responses simultaneously. The optimum conditions were determined as $9.0 \%(\mathrm{w} / \mathrm{w})$ alginate solution, $2.0 \%(\mathrm{w} / \mathrm{v}) \mathrm{CaCl}_{2}$, and $10 \mathrm{~min}$ in the gelation solution. Further investigation is required to determine the release profile of the ACNs from the particles and the stability of the encapsulated ACNs under adverse conditions.

\section{Acknowledgments}

The authors acknowledge the National Council for Research and Development (CNPq - Brazil) and the Natural Sciences and Engineering Research Council (NSERC - Canada) for financial support. 


\section{References}

[1] Castañeda-Ovando A, Pacheco-Hernández ML, Páez-Hernández ME, Rodríguez JA, Galán-Vidal CA. Chemical studies of anthocyanins: A review. Food Chem. 2009;113:859.

[2] Roy S, Khanna S, Alessio HM, Vider J, Bagchi D, Bagchi M, Sen CK. Anti-angiogenic property of edible berries. Free Radic Res. 2002;36:1023.

[3] Cvorovic J, Tramer F, Granzotto M, Candussio L, Decorti G, Passamonti S. Oxidative stress-based cytotoxicity of delphinidin and cyanidin in colon cancer cells. Arch Biochem Biophys. 2010;501:151.

[4] Jennings A, Welch AA, Spector T, Macgregor A, Cassidy A. Intakes of anthocyanins and flavones are associated with biomarkers of insulin resistance and inflammation in women. J Nutr. 2014;144:202.

[5] Gordillo G, Fang H, Khanna S, Harper J, Phillips G, Sen CK. Oral administration of blueberry inhibits angiogenic tumor growth and enhances survival of mice with endothelial cell neoplasm. Antioxid Redox Signal. 2009;11:47.

[6] Vuong T, Benhaddou-Andaloussi A, Brault A, Harbilas D, Martineau LC, Vallerand D, et al. Antiobesity and antidiabetic effects of biotransformed blueberry juice in KKA(y) mice. Int J Obes. 2009;33:1166.

[7] Stull AJ, Cash KC, Johnson WD, Champagne CM, Cefalu WT. Bioactives in blueberries improve insulin sensitivity in obese, insulin-resistant men and women. J Nutr. 2010;140:1764.

[8] Devareddy L, Hooshmand S, Collins JK, Lucas EA, Chai SC, Arjmandi BH. Blueberry prevents bone loss in ovariectomized rat model of postmenopausal osteoporosis. J Nutr Biochem. 2008;19:694.

[9] Krikorian R, Shidler MD, Nash TA, Kalt W, Vinqvist-Tymchuk MR, Shukitt-Hale B, Joseph JA. Blueberry supplementation improves memory in older adults. J Agric Food Chem. 2010;58:3996.

[10] Celli GB, Ghanem A, Brooks MS. Haskap berries (Lonicera caerulea L.) - A critical review of antioxidant capacity and health-related studies for potential value-added products. Food Bioprocess Technol. 2014;7:1541.

[11] Khattab R, Celli GB, Ghanem A, Brooks MS. Effect of thawing conditions on polyphenol content and antioxidant activity of frozen haskap berries (Lonicera caerulea L.). Curr Nutr Food Sci. 2015;11:223.

[12] Bors B, Thomson J, Sawchuk E, Reimer P, Sawatzky, R, Sander T. Haskap breeding and production - Final report. Regina: Saskatchewan Agriculture; 2012.

[13] Giusti MM, Wrolstad RE. Acylated anthocyanins from edible sources and their applications in food systems. Biochem Eng J. 2003;14:217.

[14] Hurtado NH, Morales AL, González-Miret ML, Escudero-Gilete ML, Heredia FJ. Colour, pH stability and antioxidant activity of anthocyanin rutinosides isolated from tamarillo fruit (Solanum betaceum Cav.). Food Chem. 2009;117:88.

[15] Hellström J, Mattila P, Karjalainen R. Stability of anthocyanins in berry juices stored at different temperatures. J Food Compos Anal. 2013;31:12.

[16] Rein M. Copigmentation reactions and color stability of berry anthocyanins. Dissertation - Department of Applied Chemistry and Microbiology, University of Helsinki; 2005.

[17] Matsufuji H, Kido H, Misawa H, Yaguchi J, Otsuki T, Chino M, et al. Stability to light, heat, and hydrogen peroxide at different pH values and DPPH radical scavenging activity of acylated anthocyanins from red radish extract. J Agric Food Chem. 2007;55:3692.

[18] Wrolstad RE. Anthocyanin pigments - bioactivity and coloring properties. J Food Sci. 2004;69:C419.

[19] Garzón GA, Wrolstad RE. The stability of pelargonidin-based anthocyanins at varying water activity. Food Chem. 2001;75:185.

[20] Skrede G, Wrolstad RE, Lea P, Enersen G. Color stability of strawberry and blackcurrant syrups. J Food Sci. 1992;57:172.

[21] Betoret E, Betoret N, Vidal D, Fito P. Functional foods development: Trends and technologies. Trends Food Sci Technol. 2011; 22:498.

[22] Celli GB, Ghanem A, Brooks MS. Bioactive encapsulated powders for functional foods - a review of methods and current limitations. Food Bioprocess Technol. 2015;8:1825.

[23] Zuidam NJ, Shimoni E. Overview of microencapsulates for use in food products or processes and methods to make them. In: Zuidam NJ, Nedovic VA, editors. Encapsulation Technologies for Active Food Ingredients and Food Processing. New York: Springer; 2010; pp. 3.

[24] Homayouni A, Azizi A, Ehsani MR, Yarmand MS, Razavi SH. Effect of microencapsulation and resistant starch on the probiotic survival and sensory properties of synbiotic ice cream. Food Chem. 2008;111:50.

[25] Rascón MP, Beristain CI, García HS, Salgado MA. Carotenoid retention and storage stability of spray-dried encapsulated paprika oleoresin using gum Arabic and soy protein isolate as wall materials. LWT-Food Sci Technol. 2011;44:549.

[26] Karathanos VT, Mourtzinos I, Yannakopoulou K, Andrikopoulos NK. Study of the solubility, antioxidant activity and structure of inclusion complex of vanillin with $\beta$-cyclodextrin. Food Chem. 2007;101:652.

[27] Martin KR, Appel CL. Polyphenols as dietary supplements: A double-edged sword. Nutr Diet. Suppl. 2010;2:1. 
[28] Pegg RB, Shahidi F. Encapsulation and controlled release in food preservation. In: Rahman MS, editor. Handbook of food preservation. New York: Marcel Dekker, Inc.; 1999; pp. 611.

[29] Almeida-Doria RF, Regitano-D'Arce, MA. Antioxidant activity of rosemary and oregano ethanol extracts in soybean oil under thermal oxidation. Food Sci Technol. 2000;20:197.

[30] King AH. Flavor encapsulation with alginates. In: ACS symposium series; 1988, pp. 122.

[31] Musa KH, Abdullah A, Jusoh K, Subramaniam V. Antioxidant activity of pink-flesh guava (Psidium guajava L.): Effect of extraction techniques and solvents. Food Anal Methods. 2011;4:100.

[32] Santos DT, Albarelli JQ, Beppu MM, Meireles MAA. Stabilization of anthocyanin extract from jabuticaba skins by encapsulation using supercritical $\mathrm{CO}_{2}$ as solvent. Food Res Int. 2013;50:617.

[33] Ma N, Xu L, Wang Q, Zhang X, Zhang W, Li Y, et al. Development and evaluation of new sustained-release floating microspheres. Int J Pharm. 2008;358:82.

[34] Derringer G, Suich R. Simultaneous optimization of several response variables. J Qual Technol. 1980;12:214.

[35] Giusti M, Wrolstad RE. Anthocyanins. Characterization and measurement with UV-visible spectroscopy. In: Wrolstad RE, editor. Current protocols in food analytical chemistry. New York: John Wiley \& Sons; 2001, pp. 1.

[36] Adjé F, Lozano YF, Lozano P, Adima A, Chemat F, Gaydou EM. Optimization of anthocyanin, flavonol and phenolic acid extractions from Delonix regia tree flowers using ultrasound-assisted water extraction. Ind Crops Prod. 2010;32:439.

[37] Kuo CK, Ma PX. Ionically crosslinked alginate hydrogels as scaffolds for tissue engineering: Part 1. Structure, gelation rate and mechanical properties. Biomaterials. 2001;22:511.

[38] Martinsen A, Skjåk-Bræk G, Smidsrød O. Alginate as immobilization material: I. Correlation between chemical and physical properties of alginate gel beads. Biotechnol Bioeng. 1989;33:79.

[39] Lotfipour F, Mirzaeei S, Maghsoodi M. Evaluation of the effect of $\mathrm{CaCl}_{2}$ and alginate concentrations and hardening time on the characteristics of Lactobacillus acidophilus loaded alginate beads using response surface analysis. Adv Pharm Bull. $2012 ; 2: 71$.

[40] Mandal S, Kumar SS, Krishnamoorthy B, Basu SK. Development and evaluation of calcium alginate beads prepared by sequential and simultaneous methods. Braz J Pharm Sci. 2010;46:785.

[41] Brouillard R, Delaporte B. Mechanisms of the structural transformations of anthocyanins in acidic media. J Am Chem Soc. 1977;99:1359.

[42] Jiang Z, Zhang Y, Li J, Jiang W, Yang D, Wu H. Encapsulation of $\beta$-glucuronidase in biomimetic alginate capsules for bioconversion of baicalin to baicalein. Ind Eng Chem Res. 2007;46:1883.

[43] Klokk TI, Melvik JE. Controlling the size of alginate gel beads by use of a high electrostatic potential. J Microencapsul. 2002;19:415.

[44] Liu XD, Bao DC, Xue WM, Xiong Y, Yu WT, Yu XJ, et al. Preparation of uniform calcium alginate gel beads by membrane emulsification coupled with internal gelation. J Appl Polym Sci. 2003;87:848.

[45] Deladino L, Anbinder PS, Navarro AS, Martino MN. Encapsulation of natural antioxidant extract from Ilex paraguariensis. Carbohydr Polym. 2008;71:126.

[46] Shu XZ, Zhu KJ. The release behavior of brilliant blue from calcium-alginate gel beads coated by chitosan: The preparation method effect. Eur J Pharm Biopharm. 2002;53:193. 